\title{
Management of a Smart Grid with Controlled-Delivery of Discrete Power Levels
}

\author{
Roberto Rojas-Cessa, Yifei Xu, and Haim Grebel \\ Department of Electrical and Computer Engineering \\ New Jersey Institute of Technology \\ Newark, NJ 07102 \\ \{rojas, yx63, grebel\}@njit.edu
}

\begin{abstract}
The present electrical grid uses two properties for the distribution of electrical power: perpetually energized and with discretionary access to power amounts. These two properties, although functional and practical, expose the grid to overload and consumption forecasting uncertainty. In addition, the distribution of electricity may be difficult to manage; sudden demand surge or supply shortage may ultimately lead to failures.

To overcome this, we adopt a recently proposed approach whereby electricity is delivered in discrete amounts, through what is named here as a controlled-delivery power grid. In this grid, the customer's addresses are embedded in the electrical signal, and smart loads are used to limit the supplied electrical power. These smart loads not only control the consumed amount of power but also provide reactive stability to the distribution loop. We discuss an architecture of the controlled-delivery power grid and analyze a management scheme for the distribution of power, where the capacity of the distribution loop is capped to the average of the requested power, adopting a round-robin schedule of power deliverance. We show that the efficiency of power distribution satisfies over $98 \%$ of the power requests in such scenarios of energy scarcity.
\end{abstract}

\section{INTRODUCTION}

Minimizing the difference between production and supply of electrical power and keeping the grid stable are major operation tasks for the present power grid. Recently, a controllable-delivery power grid concept, called the digital grid, has been proposed [1]; here energy is delivered in discrete (digital) levels. By contrast, present grids distribute energy in a continuous fashion. In analog grids, generators might be adapted to re-balance the grid at times of overproduction and safety margins are imposed to avoid under production.

At times of overproduction, whether because of low demand or over production caused by discrepancies between forecasted and actual power demand, the electricity generators might be slowed down to re-balance the grid. Underproduction may be avoided by providing a safety margin on the amount of electricity generated.

The demand forecast includes a careful data analysis and predictive models of consumption levels during each time of the day and each day of the year, and modified by extenuating circumstances. Therefore, the demand-supply stability is mostly based on keeping safety margins in the form of over production [2].
Everyday experience shows us that the power grid is fairly robust; yet, the response of power providers (and generators) may still lag after variations in consumers' demand. In real time, suppliers may monitor the amount of power supplied and consumed only on a large-scale basis (namely, for circuit loops containing a large number of customers). An underlying assumption on presently used systems is that fluctuations of local consumption, especially local power surges, are rapidly averaged out when the number and variety of consumers is large.

A tighter grid stability may increase the efficiency of the grid, and in turn, extend the life of non-renewable energy resources. To achieve this high degree of stability, energy could be supplied in discrete amounts, as energy quanta, to customers in what we call a controlled-delivery grid [1], [3]. In this grid, energy may be directed to specific customer(s) and the delivered amounts are determined and controlled. Two properties of the present grid, perpetually energized and discretionary access make it a challenge to follow. The perpetually energized property allows customers to access and consume electricity at any time. The discretionary access property allows customer to demand discretionary amounts of power, whose power capacity may be limited. An example of the benefits of a controlled-delivery grid is the case of grid overload. Here the load, and therefore, the amount of drained power cannot be limited nor controlled, potentially putting the grid segment at risk of failure. Current alternatives to minimize failures is the shutting down of grid segments, and this makes the monitoring the state of the grid a key aspect. Close monitoring of the grid's performance may be achieved by deploying parallel (auxiliary) sensing networks [4]-[13]. Concerns about ensuring working paths, which are perpetually energized translate into additional management complexity [11], [14]. Therefore, the adoption of a controlled distribution of power, which is seamlessly coupled with grid monitoring, becomes an important part of grid management.

In a controlled-delivery grid, customers issue requests for needed power. The provider may fully or partially grant the request, however, within allowable discrete levels. Such an approach would facilitate an estimation of total users' demand and giving the provider the ability to determine how to satisfy 
the requests before fulfilling them. This model also implies the adoption of a controlled supply. The discretionary access property of the current grid challenges the deployment of a controlled-supply model. The present grid is expected to provide almost limitless power, which encourages customers to indiscriminately connect large loads. However, such availability of power also puts the grid at risk for line overloads and disables the grid from distributing electrical power under cases of shortage.

The amount of delivered power can be controlled with the designation of ownership and limited to specific power levels. Here, ownership means that electric power may only be used by the destined customer(s). To achieve this, the electrical signal carries the customer address and equipment at the customer's premises access power if the address carried in the signal matches the customer's address [1]. The address of such a customer can be carried by the electrical signal or by an auxiliary data network.

The concept of controlling the distribution of energy through micro-grids as the next generation electrical grid has been discussed before [15]. Approaches to verify customers identification with an address before the start of an energy transmission can be applied to determine the electricity path [11]. However, the one-to-one interconnection required by these approaches limits its scalability, in terms of the number of connected customers. Some of these works are motivated by the consideration of alternative-energy sources, where sources and appliances can be matched through dedicated lines, using direct current (DC) multiplexors [16].

Providing a means to control the amount of power delivered to a user is needed to avoid excessive loads. With a controlled delivery, a demand-supply mechanism can be used to manage not only the power supply, but also to manage the grid more efficiently. In this case, a request is made to the power provider for a precise power demand at the time a switch of an electrical device is turned ON. A hand-shake protocol is established between the consumer and supplier, and power is delivered to the consumer (and perhaps to a particular appliance). To enable controllability on the amount of power that a user takes from the grid, a defined supply time and smart current limiters can be adopted.

The distribution mechanism used by the controlled-delivery grid determines its efficiency. We adopt a round-robin schedule for the selection of customers in the distribution of limited amounts of power. A round-robin schedule provides fairness of service among all customers since it follows a predetermined list; the last serviced customer has the lowest priority for receiving new service. We use this scheme to estimate the level of satisfied energy requests.

We modeled and studied a controlled-delivery power grid with a moderate number of customers, and show that the studied approach can accommodate over $98 \%$ of power requests when we cap the maximum power supply to the average of the power demand. In this paper, we refer to power and electrical energy indistinctly, as one can be converted onto the other

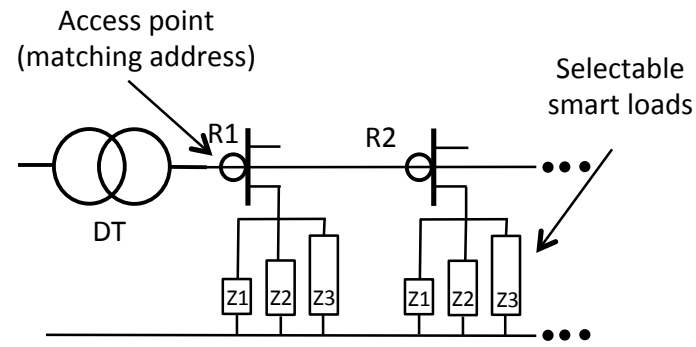

Fig. 1. Smart load selected for each customer.

with consideration of a time variable.

The remainder of this paper is organized as follows. Section II introduces the controlled-delivery power grid. Section III analyses the achievable satisfaction of energy requests for a power loop. Section IV presents our conclusions.

\section{Controlled-Delivery POWER GRID}

The power grid with controlled delivery aims at supplying discrete and finite amounts of energy to customers. The approach minimizes the difference between energy generation and demand, facilitates power distribution amongst several grids, and increases grid stability through local and instantaneous traffic monitoring.

The controlled-delivery power grid carries energy in discrete units, or energy quanta. Each quantum unit of power may be controlled by time, current, or a combination of both. The electrical signal carries the destination address(es) of specific (or multiple) customer(s) who are the only one(s) allowed to get access to the transmitted energy. The destination address may be embedded into the electrical signal. Because energy may need to simultaneously distributed to a single or a large number of customers, addressing through code division multiple access (CDMA) [17] or a modulated signal could be employed. The amount of energy per slot may be scaled up in two dimensions: 1) by using several time slots together and form a train of granted requests, and 2) by setting the amount of energy transmitted within a time slot by adjusting the amount of current. In this power grid model, the amount of current may be set to discrete levels. The selected level of power destined for a customer may be controlled by selective current limiters, called smart loads, at the customer premises, as Figure 1 shows. The energy supplier performs the selection of a smart load by also embedding the amount of current granted per customer in the electrical signal.

The distribution loop (Figure 2) has a large number of customers. Each of them is able to receive addressed energy and paired with controlled smart loads. Nodes of the controlleddelivery grid (labeled as DT in the figure) adjust the voltage levels, perform signal conversion, and forward the embedded addresses to the signal forwarded to the end customers (labeled as R1 to R50 in the figure).

The path of data, coupled to the power grid: a) finds the requested energy levels as issued by customers (or local 


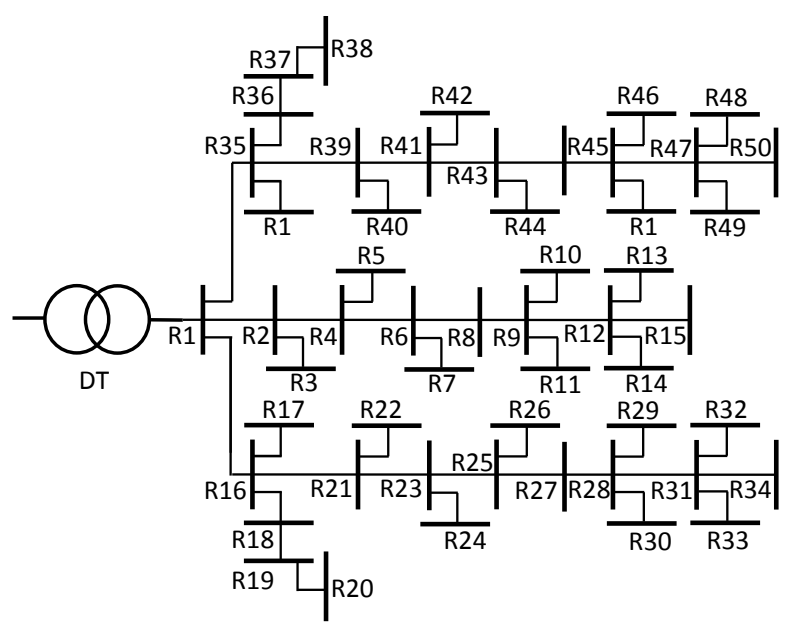

Fig. 2. Distribution network.

distribution points) and assigns the incoming power to supply those requests, b) finds routing information about where to forward the energy, and c) attaches the destination address and the amount of current for the supplied power for secure and guaranteed delivery. Because energy quanta can be used for each destination (in terms of a single unit), the supply of energy does not need to differentiate the incoming energy (from the generator) but the outgoing one, in a destination (or customer) basis.

\section{A. Nodes for the Controlled-delivery Power Grid}

In this paper, we adopt a shared distribution loop where energy is targeted to specific customers by appending the customers' grid address to the energy, which are called here energy packets. Distribution points or nodes of the grid, forward energy packets to the end-customer after determining the destined address, which is provided by the local distribution substations. The array of nodes along the distribution segment receive the energy packets, decode the addresses and forward the packets to the proper destinations. This equipment is tentatively and coarsely divided into three categories: grid router, power switch, and power access point, in alignment with the different devices used in a data network. Figure 3 shows the different components in their placement at various part of the grid.

The grid equipment of this grid represents a fusion of power electronics and computer networks, where power electronics are used to transmit energy packets to destined customers (through the local distribution grid). The power path of the switches must enable routing a energy from inputs to the outputs but at different discrete current levels.

\section{B. Grid Routers}

Grid routers are mainly connected in the core and regional segments. In addition to participating in carrying energy from the generation plants towards the customers, they also

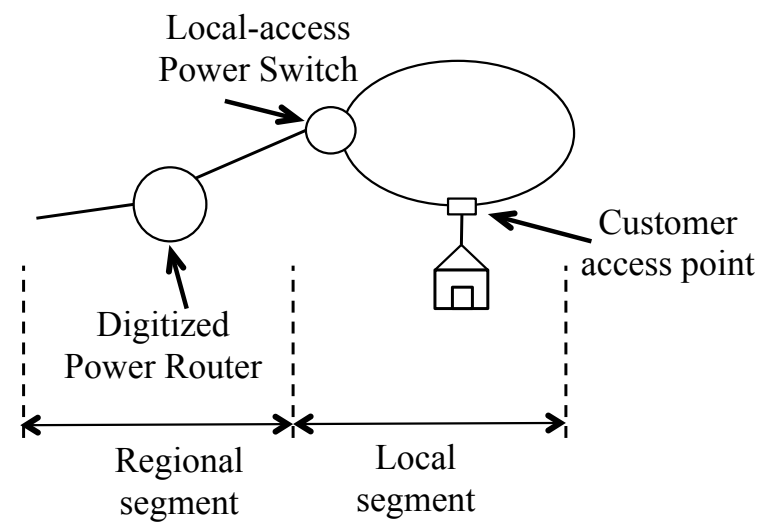

Fig. 3. Different interconnection equipment in the controlled-delivery power grid.

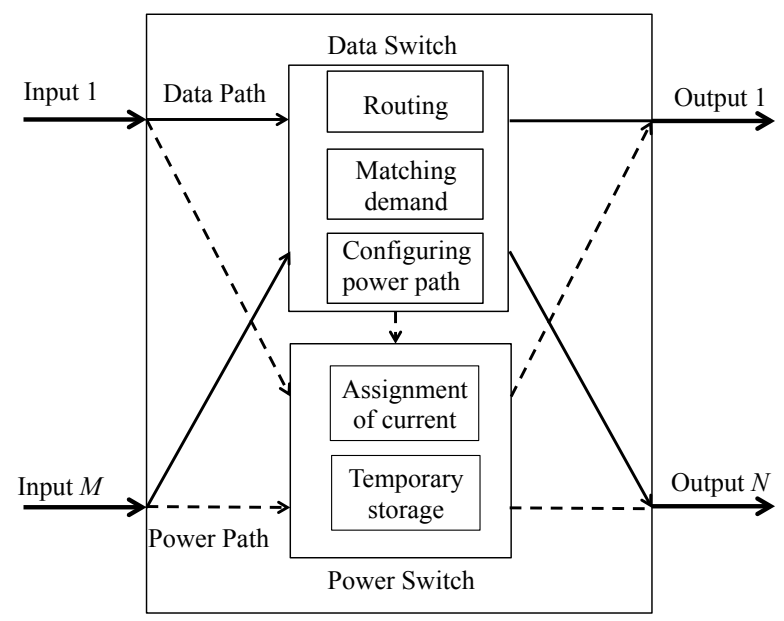

Fig. 4. Architecture of a power switch.

interconnect segments from local loops under different administrations, with the purpose to route energy to alternative local segment or from different generation plants. They perform routing between these two ends, energy sources and customers.

Figure 4 shows a possible architecture of a grid router. The switch capacity and size depends on its location in the digital grid.

The grid routers comprise a power path, through which power is delivered to the customers, and a data path, which transmits the information to monitor customer demand and supply and to configure the power path of the switches. The inputs and outputs of a grid switch receive data and energy packets, which are separated in the switch for configuration, and these two channels are put joined at the egress side of the switch. The inputs and outputs could use a shared or dedicated transmission lines. A shared line means that the high voltage to transmit energy carries the data information embedded in it. Dedicated lines means that energy and data use one line separately (e.g., a data line could be implemented with an optical fiber). 


\section{Power Switch}

A power switch may be used to interface local and regional segments. It works similar to a grid router, with data and power paths, however, the routing functions are limited as it is mostly used as the access node to local loops. The power switch aggregates energy request from local segments and forwards them to grid routers. As energy packets flow into the power switch, the energy is forwarded to the local segments. An example of a possible location of a power switch is shown in Figure 3. Power switches have a switching control and a power distributor. These switches are sometimes called intelligent power switches because of their application in smart grids [9], [14]. However, as the number of customer increases, so does the complexity of the switch management functions, including the power [18] and data sections of the switch [19].

\section{Power Access Point}

A power access point (PAP) is used by a customer to access the digital grid. The PAP collects requests of energy. The PAP also communicates with the power switch (at the substation) to request energy and direct the energy to the customer; it acknowledges and monitors the received energy packets. The PAP is also the security device used by the provider as it controls the amount of current drawn by a customer (some approaches to protect circuits from circuit overload on continuos wave signals have been considered cost effective [20]). The PAP is the boundary of the network under the administration of the energy provider. Figure 3 shows a PAP, interconnecting the customer to the local loop.

\section{E. Distribution Points}

Distribution points, which may be implemented by grid routers or power switches, transfer energy in two possible directions: 1) from the generator to the customer, and 2) from the distribution loop towards other grids (energy re-route). These distribution points also perform energy conversions, including voltage step-up and -down, as they may also be used to interconnect different distribution segments, from generators to costumer supply. The forwarding of energy to other grids is out of the scope of this paper.

The forwarding functions of a distribution point include aggregation of energy packets that need to be sent down to the distribution loop, and distribution of energy (which is the case when an incoming energy packet is split into two or more energy packets, as Figure 5 shows, sent through different ports of the grid router). However, for the grid router to perform these functions, a forwarding table needs to be built and table lookup needs to be performed [21]. Table lookup has the special requirement to be performed in shorter time that it takes to transmit an energy packet.

As the objective is on supplying energy in discrete levels, a distribution point may have different set of transformers that support different levels of current rather than having a transformer to support a single maximum amount of current, and these are coupled with the smart loads at the customer side.

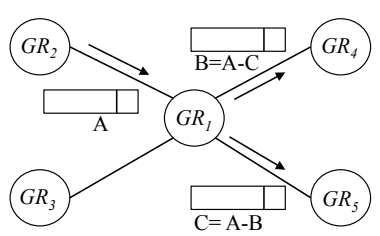

(a) Example of splitting an energy packet.

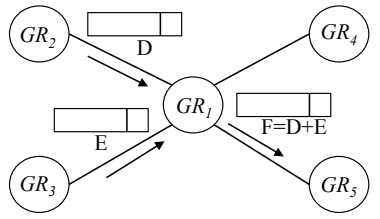

(b) Example of aggregating energy packets.
Fig. 5. Examples of splitting and aggregation by distribution points.

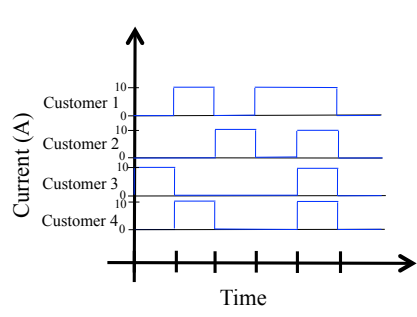

(a)

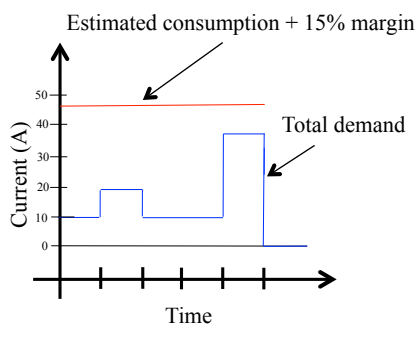

(b)
Fig. 6. Example of current demand (a) individual demand of four customers, and (b) total demand.

These levels of current provide the different amounts required to satisfy the aggregated amount of current as requested by customers and as granted by the distribution point.

Figure 6 shows an example of how the energy is supplied to demanding customers, considering 1-level requests and a safety margin needed to maintain a grid stable. There are four customers, each issuing a random request for an amount of current. The grid can only provide discrete levels of current, which are 1, 2, 4, or 8 Amps. Figure 6(a) shows the individual requests and Figure 6(b) shows the total current supplied, which is within the maximum loop capacity.

\section{F. Distribution Management Scheme using Round-Robin Se- lection}

As the distribution loop has limited capacity, the aggregated customers' requests may exceed it. The distribution point selects requesting customers in a round-robin schedule for satisfying the discrete-amount requests. The selection performed by the distribution point is described as follows:

Phase 1. Each customer issues an energy request, if any, to the distribution point.

Phase 2. The distribution point grants a request if the amount of energy remaining is larger than the requested level (full supply), or if the remaining energy is equal to smaller level or energy (partial supply). Once the remaining amount of energy is zero or smaller than the smallest level, no more customer requests are granted, and the distribution point waits until the next cycle. Energy is supplied in the following time slot after a request is granted.

\section{Evaluation of ENERgy Distribution}

We evaluated the efficiency of the management mechanisms for the controlled-delivery power grid. For this, we modeled 
a distribution loop and the management mechanisms for computer simulation on Matlab. The energy requests of a customer were modeled as a two-state (ON-OFF) modulated Markov process. The energy request that is currently OFF becomes ON with probability $q$, or remains OFF with a probability $1-q$ for one hour, which is a characteristic time window. The request change its state to OFF with a probability $p$, or continued its $\mathrm{ON}$ state with probability $1-p$. The ratio of satisfied customers, defined as customers whose energy request is fully satisfied was then assessed. In addressing a request the program first fulfilled the lower power needs of all customers and moved on to fulfill the next level using the round-robin rule. Specifically, the distribution node keeps track of the customers served as long as there were non-served customers remaining. We considered distribution loops with 10, 100, and 1000 customers. The demand from all customers in the loop was averaged for 1 year (365 days) and the value obtained was used to define the capacity of the loop. Each day was split into three 8-hour intervals, with each interval having a different average burst size, with values of 4,6 , and 3 hours. This approach is very similar to the way power grid providers estimate production levels [22].

Table I shows the 12 different scenarios (enumerated from 1 to 12 ) considered in our study. The scenarios differ in the number possible levels of energy, amount of energy per level that a customer may request, and the number of customers considered. The controlled-delivery power grid then attempts to satisfy the energy requested. However, if the power grid cannot fully provide for an energy request (e.g., because of limited energy capacity), it attempts to provide the next lower energy level instead. For example, Scenario 2 calls for four energy levels: 3, 6, 9 and 12 units. If a request is made for 6 units and the limited capacity grid is not able to fulfill such request, it may provide 3 units instead. Note that this scenario cannot be accommodated in the current power grid infrastructure as energy is not addressable and all customers connected would consume whatever is available from the distribution line, leaving most customers unsatisfied.

TABLE I

TEST SCENARIOS

\begin{tabular}{c|c|c|c}
\hline Scenario & $\begin{array}{c}\text { No. of } \\
\text { customers }\end{array}$ & Levels & Prob. per level \\
\hline \hline 1 & 10 & $3,6,9,12$ & $0.1,0.2,0.3,0.4$ \\
\hline 2 & 100 & $3,6,9,12$ & $0.1,0.2,0.3,0.4$ \\
\hline 3 & 1000 & $3,6,9,12$ & $0.1,0.2,0.3,0.4$ \\
\hline 4 & 10 & $1,2,4,8$ & $0.1,0.2,0.3,0.4$ \\
\hline 5 & 100 & $1,2,4,8$ & $0.1,0.2,0.3,0.4$ \\
\hline 6 & 1000 & $1,2,4,8$ & $0.1,0.2,0.3,0.4$ \\
\hline 7 & 10 & $3,6,9,12,15,18,21,24$ & $0.025,0.05,0.075,0.1,0.125,0.15,0.175,0.3$ \\
\hline 8 & 100 & $3,6,9,12,15,18,21,24$ & $0.025,0.05,0.075,0.1,0.125,0.15,0.175,0.3$ \\
\hline 9 & 1000 & $3,6,9,12,15,18,21,24$ & $0.025,0.05,0.075,0.1,0.125,0.15,0.175,0.3$ \\
\hline 10 & 10 & $1,2,4,8,16,32,64,128$ & $0.025,0.05,0.075,0.1,0.125,0.15,0.175,0.3$ \\
\hline 11 & 100 & $1,2,4,8,16,32,64,128$ & $0.025,0.05,0.075,0.1,0.125,0.15,0.175,0.3$ \\
\hline 12 & 1000 & $1,2,4,8,16,32,64,128$ & $0.025,0.05,0.075,0.1,0.125,0.15,0.175,0.3$ \\
\hline
\end{tabular}

We consider four different discrete levels of energy with discrete levels with linear (Scenarios 1 to 3) and exponential (Scenarios 4 to 6) step increase, and eight discrete levels, also with linear (Scenarios 7 to 9) and exponential (Scenarios 10 to 12) step increase. For other Scenarios, please refer to [3]. Furthermore, the customer energy request is issued for a level requested by an assigned probability. The table then shows the probabilities for each possible energy level in each scenario, in the third column.

TABLE II

RATIO OF SATISFIED REQUESTS FOR THE DIFFERENT TEST SCENARIOS

\begin{tabular}{c|c|c}
\hline Scenario & $\begin{array}{c}\text { Satisfaction } \\
\text { ratio (\%) }\end{array}$ & $\begin{array}{c}\text { Outstanding } \\
\text { energy (\%) }\end{array}$ \\
\hline \hline 1 & 76.90 & 23.3 \\
\hline 2 & 94.68 & 5.3 \\
\hline 3 & 98.64 & 1.4 \\
\hline 4 & 72.91 & 27.4 \\
\hline 5 & 93.69 & 6.3 \\
\hline 6 & 98.45 & 1.6 \\
\hline 7 & 76.62 & 23.6 \\
\hline 8 & 94.41 & 5.6 \\
\hline 9 & 98.47 & 1.5 \\
\hline 10 & 57.45 & 43.1 \\
\hline 11 & 87.83 & 12.3 \\
\hline 12 & 96.42 & 3.6 \\
\hline
\end{tabular}

Table II shows the ratio of satisfied customer requests (satisfaction ratio) for all cases. The table shows the satisfaction ratio and the outstanding energy percentage. We summarize the results as follows. The satisfaction ratio increases as the number of customer increases, from $76.9 \%$ to $98.68 \%$ for four discrete levels with linear increase. Similar increase is observed for discrete levels with exponential distributions. We also observe that increasing the number of levels may not be necessary. Take for example, the maximum satisfaction ratio of a scenario with eight levels with linear increase of discrete levels (Scenario 9) is similar to that of a scenario with four levels (Scenario 3). The scenarios with exponential increase show similar trend.

We also estimated the level of outstanding energy, which is the percentage of energy that is remained to be supplied. The outstanding energy for 1000 customers for different levels of energy changes from 1.4 to $3.6 \%$, as the number of energy levels increases. These values may also be concurred by the observations made from analyzing the satisfaction ratio.

Figure 7 shows the percentage of outstanding energy for fully satisfied requests. The figure shows the decrease in the percentage of outstanding energy as the number of customers increases.

\section{CONCLUSiOnS}

In meeting the challenges of the modern power grid where many providers and customers share the same transmission lines, we studied a grid model where energy is supplied in discrete amounts. This power grid presents an alternative to the present grid, which is a) perpetually energized and 2) discretionary accessed.

In this controlled-delivery power grid, energy is delivered in quantum units, e.g., discrete current levels while keeping the voltage constant. In this grid, electrical power is addressable to single or multiple customers, and the amount of energy delivered to a specific customer is bound by smart loads. The 


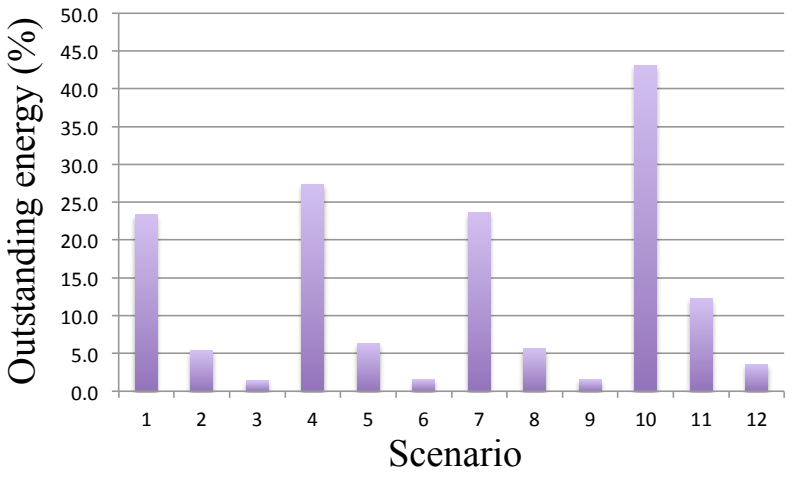

Fig. 7. Outstanding energy for the considered Scenarios.

customer and supplier then follow a request-supply communication protocol and the supplier can then manage energy grants. Since the power demand is dynamic and necessitates sharing of grid lines, the energy is delivered asynchronously among all customers.

We adopted a round-robin scheme for selecting the customer(s) for supplying energy in a distribution loop when energy is limited. We evaluated the management scheme by looking at the ratio of satisfied energy requests. We simulated customer request needs over one year, and showed that an efficient power delivery system requires a very small amount of energy capacity to satisfy $98 \%$ or more of customer energy requests. We show that the distribution of discrete energy levels is scalable as: 1) the grid achieves a high satisfaction ratio for a large number of customers and 2) a moderate number of energy levels is enough to achieve these satisfaction ratios.

\section{REFERENCES}

[1] Y. Xu, R. Rojas-Cessa, and H. Grebel, "Allocation of discrete energy on a cloud-computing datacenter using a digital power grid," in Green Computing and Communications (GreenCom), 2012 IEEE International Conference on. IEEE, 2012, pp. 615-618.

[2] S. Pahwa, A. Hodges, C. Scoglio, and S. Wood, "Topological analysis of the power grid and mitigation strategies against cascading failures," in Systems Conference, 2010 4th Annual IEEE, april 2010, pp. 272 -276.

[3] R. Rojas-Cessa, Y. Xu, and H. Grebel, in Electrical Power and Energy Conference. IEEE, 2013, pp. 1-5.

[4] R. Gono, S. Rusek, and M. Kratky, "Reliability analysis of distribution networks," in Electrical Power Quality and Utilisation, 2007. EPQU 2007. 9th International Conference on, oct. 2007, pp. $1-5$.

[5] H. He, "Toward a smart grid: Integration of computational intelligence into power grid," in Neural Networks (IJCNN), The 2010 International Joint Conference on, july 2010, pp. 1 -6.

[6] S. Galli, A. Scaglione, and Z. Wang, "For the grid and through the grid: The role of power line communications in the smart grid," Proceedings of the IEEE, vol. 99, no. 6, pp. 998 -1027, june 2011.

[7] W.-H. Liu, K. Liu, and D. Pearson, "Consumer-centric smart grid," in Innovative Smart Grid Technologies (ISGT), 2011 IEEE PES, jan. 2011, pp. $1-6$.

[8] K. Budka, J. Deshpande, J. Hobby, Y.-J. Kim, V. Kolesnikov, W. Lee, T. Reddington, M. Thottan, C. White, J.-I. Choi, J. Hong, J. Kim, W. Ko, Y.-W. Nam, and S.-Y. Sohn, "Geri - bell labs smart grid research focus: Economic modeling, networking, and security amp; privacy," in Smart Grid Communications (SmartGridComm), 2010 First IEEE International Conference on, oct. 2010, pp. $208-213$.
[9] G. Lu, D. De, and W.-Z. Song, "Smartgridlab: A laboratory-based smart grid testbed," in Smart Grid Communications (SmartGridComm), 2010 First IEEE International Conference on, oct. 2010, pp. $143-148$.

[10] W.-Y. Yu, V.-W. Soo, M.-S. Tsai, and Y.-B. Peng, "Coordinating a society of switch agents for power distribution service restoration in a smart grid," in Intelligent System Application to Power Systems (ISAP), 2011 16th International Conference on, sept. 2011, pp. 1 -7.

[11] R. Abe, H. Taoka, and D. McQuilkin, "Digital grid: Communicative electrical grids of the future," Smart Grid, IEEE Transactions on, vol. 2, no. 2, pp. $399-410$, june 2011.

[12] F. Bouhafs, M. Mackay, and M. Merabti, "Links to the future: Communication requirements and challenges in the smart grid," Power and Energy Magazine, IEEE, vol. 10, no. 1, pp. 24 -32, jan.-feb. 2012.

[13] Z. Fan, P. Kulkarni, S. Gormus, C. Efthymiou, G. Kalogridis, M. Sooriyabandara, Z. Zhu, S. Lambotharan, and W. Chin, "Smart grid communications: Overview of research challenges, solutions, and standardization activities," Communications Surveys Tutorials, IEEE, vol. PP, no. 99, pp. $1-18,2012$.

[14] T. Takuno, M. Koyama, and T. Hikihara, "In-home power distribution systems by circuit switching and power packet dispatching," in Smart Grid Communications (SmartGridComm), 2010 First IEEE International Conference on, oct. 2010, pp. $427-430$.

[15] M. M. He, E. M. Reutzel, X. Jiang, R. H. Katz, S. R. Sanders, D. E. Culler, and K. Lutz, "An architecture for local energy generation, distribution, and sharing," in IEEE Energy2030, Nov. 2008, pp. 1 -6.

[16] T. Takuno, Y. Kitamori, R. Takahashi, and T. Hikihara, "Ac power routing system in home based on demand and supply utilizing distributed power sources," Energy, vol. 4, no. 5, pp. 717-726, june 2011.

[17] A. J. Viterbi et al., CDMA: Principles of spread spectrum communication. Addison-Wesley Reading, 1995, vol. 129.

[18] A. Costabeber, P. Tenti, and P. Mattavelli, "Surround control of distributed energy resources in micro-grids," in Sustainable Energy Technologies (ICSET), 2010 IEEE International Conference on, dec. 2010, pp. $1-6$.

[19] R. Rojas-Cessa, Z. Dong, and C.-B. Lin, "Coexistence of streaming and packetized data throughout the protocol stack," in Sarnoff Symposium, 2009. SARNOFF '09. IEEE, 30 2009-april 1 2009, pp. 1 -5.

[20] F. Lei, G. Ruifeng, Z. Wanrong, and Y. Xiaoping, "Type and characteristic analysis of fault current limiter," in Electric Power Equipment - Switching Technology (ICEPE-ST), 2011 1st International Conference on, oct. 2011, pp. $356-361$.

[21] R. Rojas-Cessa, L. Ramesh, Z. Dong, L. Cai, and N. Ansari, "Parallel search trie-based scheme for fast ip lookup," in Global Telecommunications Conference, 2007. GLOBECOM'07. IEEE. IEEE, 2007, pp. 210-214.

[22] S. Shao, T. Zhang, M. Pipattanasomporn, and S. Rahman, "Impact of tou rates on distribution load shapes in a smart grid with phev penetration," in Transmission and Distribution Conference and Exposition, 2010 IEEE PES. IEEE, 2010, pp. 1-6. 